\title{
Femtosecond Z-scan measurement of GaN
}

\author{
Yong-Liang Huang, Chi-Kuang Sun, ${ }^{\text {a) }}$ and Jian-Chin Liang \\ Department of Electrical Engineering and Graduate Institute of Electro-Optical Engineering, \\ National Taiwan University, Taipei 10617, Taiwan, Republic of China \\ S. Keller, M. P. Mack, U. K. Mishra, and S. P. DenBaars \\ Department of Electrical and Computer Engineering, University of California, Santa Barbara, \\ Santa Barbara, California 93106
}

(Received 30 June 1999; accepted for publication 6 October 1999)

\begin{abstract}
Nonlinear refractive index $n_{2}$ of GaN was measured for the below-band-gap ultraviolet (UV) wavelength region using Z-scan techniques with femtosecond UV pulses. A large nonlinear refractive index of $-2.9 \pm 1.2 \times 10^{-12} \mathrm{~cm}^{2} / \mathrm{W}$ was obtained at a wavelength of $368 \mathrm{~nm}$. The distribution of $n_{2}$ versus wavelength was found to be consistent with a model described by the quadratic Stark effect, which is the dominant factor contributed to the nonlinear refractive index near the band gap. Our experiments show that $\mathrm{GaN}$ is an excellent nonlinear material with large negative $n_{2}$ in the UV region. (C) 1999 American Institute of Physics. [S0003-6951(99)01948-8]
\end{abstract}

The nonlinear refractive index plays an important role in many interesting phenomena of nonlinear optics. Nonlinear materials with large optical nonlinearities and fast response speeds are required for future photonic devices such as all optical switches and modulators. GaN shows its important role in blue-emitting diodes and lasers due to its large direct band gap at $365 \mathrm{~nm}(3.39 \mathrm{eV}) .{ }^{1} \mathrm{GaN}$ material also shows its advantages as visible blind ultraviolet (UV) detectors and high-power electronics. Many experiments have been performed to study its physical and optical properties. However, its nonlinear properties are seldom reported. An exceptional large positive nonlinear refractive index of 1 $\times 10^{-12} \mathrm{~cm}^{2} / \mathrm{W}$ at $532 \mathrm{~nm}$ has been reported by a degenerate four-wave-mixing experiment in $\mathrm{GaN}$ epilayers. ${ }^{2}$

Since GaN shows its excellent properties in the blue to UV region, here we report our studies of the nonlinear refractive index of $\mathrm{GaN}$ in its below-band-gap UV region by using frequency-doubled UV pulses from a femtosecond Ti: sapphire laser. The femtosecond UV pulses provide a large peak intensity to induce third-order nonlinearity in GaN. Among all techniques measuring the third-order nonlinearity, $Z \operatorname{scan}^{3}$ is the most popular method due to its high sensitivity, simple experimental setup, and easy alignment. We applied this method in our GaN experiments. However, this technique requires a high-quality $\mathrm{TEM}_{00}$ optical Gaussian beam, a uniform sample, and a stable light source.

We performed our study on a high-quality 5 - $\mu \mathrm{m}$-thick GaN film that was grown by metal-organic chemical-vapor deposition on a double-sided polished $c$-plane sapphire substrate. After annealing the substrate and deposition of the nucleation layer, an unintentionally doped GaN layer of 5 $\mu \mathrm{m}$ thickness was grown. The absorption spectrum of this $\mathrm{GaN}$ thin film showed that the band gap was located around $365 \mathrm{~nm}(3.39 \mathrm{eV})$. By adding aluminum composition into $\mathrm{GaN}$, the band gap would be widened and the application wavelength can be further extended into the UV region.

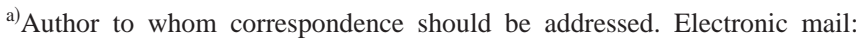
sun@cc.ee.ntu.edu.tw
}

The experiments were performed using a Kerr-lens mode-locked Ti:sapphire laser tunable between 690 and 800 $\mathrm{nm}$ wavelength with a repetition rate of $82 \mathrm{MHz}$. The output pulses of the Ti:sapphire laser were then frequency doubled using a 500- $\mu$ m-thick BBO crystal. A color filter was used to pass the second-harmonic UV pulses and to eliminate the fundamental infrared pulses. The transverse mode of the second-harmonic UV beam was the $\mathrm{TEM}_{00}$ mode. The wavelength of the UV pulses was tuned between 368 and $391 \mathrm{~nm}$ in our Z-scan measurement. A $180 \mathrm{fs}$ full width at half maximum pulse width of the frequency-doubled pulses was measured by using a two-photon absorption-type pump-probe autocorrelation in a bulk GaN sample. ${ }^{4}$ After the color filter, the measurement was then performed using the standard $Z$-scan technique with a $25 \mathrm{~mm}$ focal-length objective lens. The incident pulse energies corrected for Fresnel reflection at the front sample surface were between 0.18 and $0.32 \mathrm{~nJ}$, corresponding to pump intensities on the order of 1.3-2.3 $\mathrm{GW} / \mathrm{cm}^{2}$ assuming a beam radius of $5 \mu \mathrm{m}$. As the sample moved through the focus in the beam-propagated direction (" $Z$ ' direction), the far-field transmittance through a finite aperture was measured by a detector. Due to self-focusing or self-defocusing effects, a $Z$-scan trace with a peak and valley could thus be observed. The laser beam was chopped and the detected signal was sent to a lock-in amplifier to increase the signal-to-noise ratio. The sign of $n_{2}$ could be obtained directly from the shape of the $Z$-scan trace. The nonlinear phase change and the value of $n_{2}$ could then be easily obtained from the peak-to-valley difference of the Z-scan trace.

Surface roughness or sample nonuniformity might lead to a large variation in the background transmittance, which could mask the signal induced by the nonlinear refractive index. A low-energy scan was utilized for establishing a background signal. These parasitic effects can thus be removed by subtracting the low-energy scan from a highenergy scan. ${ }^{3}$ We have also performed our measurements on a comparison sapphire substrate and no signal was found. A typical small aperture $(S=80 \%) Z$-scan signal and open aperture background signal measured at $371.2 \mathrm{~nm}$ are shown in 

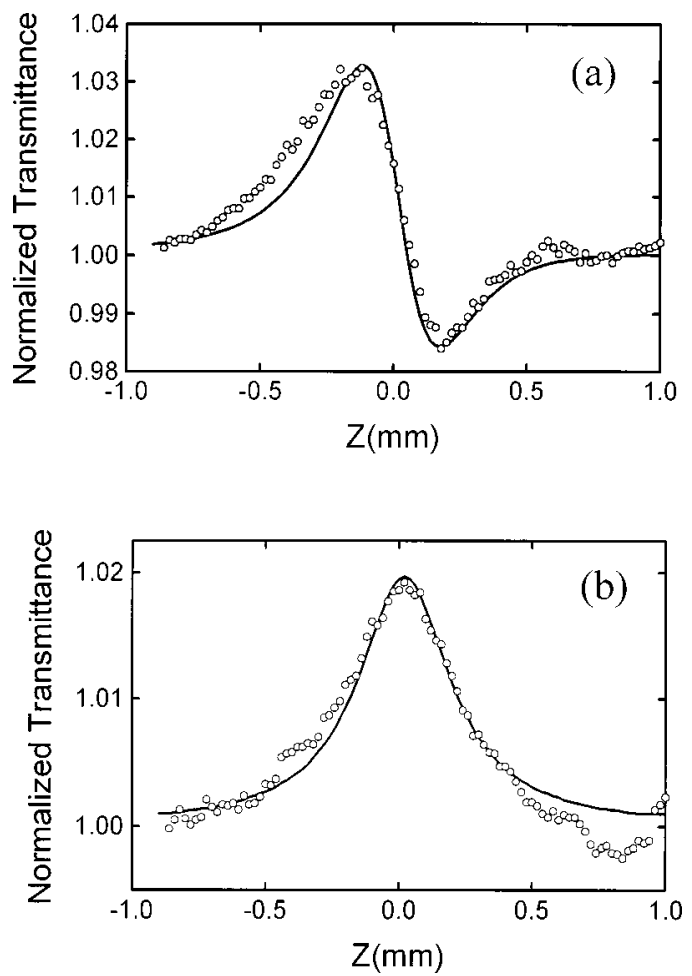

FIG. 1. (a) $Z$-scan trace (open circle) of GaN measured with a pulse energy of $0.21 \mathrm{~nJ}$ at $371.2 \mathrm{~nm}$. The solid line is a fitting curve with a beam radius of $5 \mu \mathrm{m}$. (b) Open aperture background signal (open circle) and fitting curve (solid line)

Figs. 1(a) and 1(b), respectively. The solid lines are the fitted curves with a beam radius of $5 \mu \mathrm{m}$, which agrees with a spot-size measurement with transmission through a pinhole. The measured trace shows a negative $n_{2}$. However, the peak was found to be enhanced and the valley was found to be suppressed. This behavior was an indication of absorption saturation. This absorption saturation behavior was also reflected in the open aperture traces shown in Fig. 1(b). Absorption saturation functions as an effective negative twophoton absorption coefficient $\beta .{ }^{5,6} \mathrm{~A}$ similar behavior was previously reported in an azobenzene-functionalized polymer film. ${ }^{6}$ We attribute our observed effect to the absorption saturation of $\mathrm{GaN}$ band-tail states. ${ }^{7}$ This absorption saturation behavior gradually disappeared as we tuned our pulse wavelength away from the band gap toward the visible region. Following the standard derivation procedure provided by Sheik-Bahae et al., ${ }^{3}$ a nonlinear refractive index of -1.4 $\times 10^{-12} \mathrm{~cm}^{2} / \mathrm{W}$ and a negative effective $\beta$ of $-37 \mathrm{~cm} / \mathrm{GW}$ can thus be derived from these specific $Z$-scan curves shown in Fig. 1.

$Z$-scan measurements were also performed at difference wavelengths between 368 and $391 \mathrm{~nm}$ or at different sample positions. As we tuned the laser toward longer wavelength, the measured $Z$-scan traces first became symmetric, then the peak was found to be suppressed and the valley was found to be enhanced, an indication of two-photon absorption. Figures 2(a) and 2(b) show the measured Z-scan traces at wavelengths of 375 and $387 \mathrm{~nm}$, respectively. The solid lines are fitted traces. A symmetric Z-scan trace was observed for the $375 \mathrm{~nm}$ trace while the two-photon absorption effect is reflected in the $387 \mathrm{~nm}$ trace, indicating the domination of two-photon absorption over band-tail absorption saturation
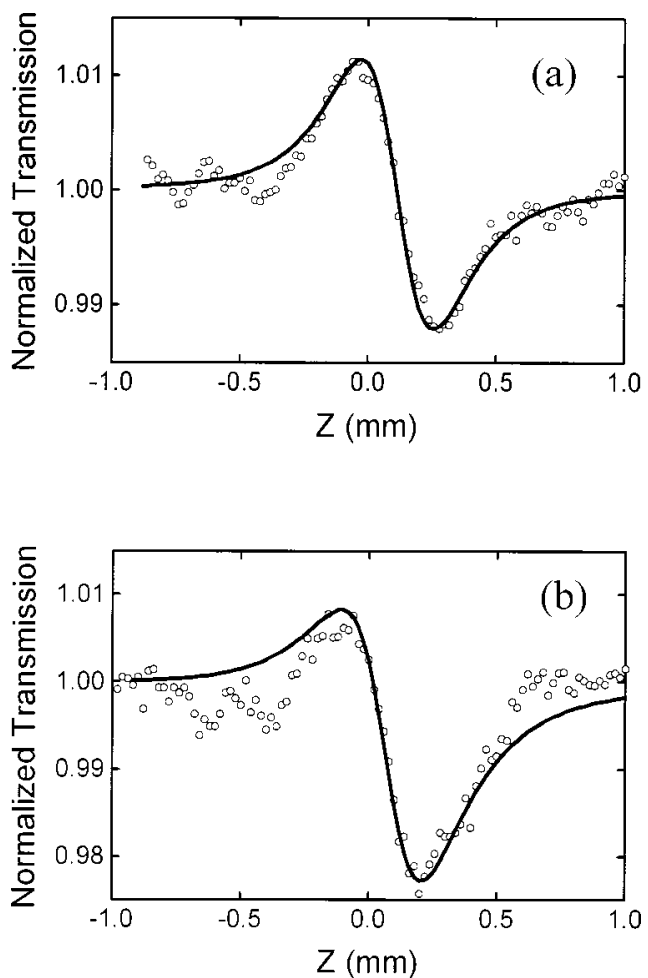

FIG. 2. (a) Z-scan trace (open circle) of GaN measured with a pulse energy of $0.29 \mathrm{~nJ}$ at $375 \mathrm{~nm}$. The solid line is a fitting curve. (b) Z-scan trace (open circle) of GaN measured with a pulse energy of $0.32 \mathrm{~nJ}$ at $387 \mathrm{~nm}$. The solid line is a fitting curve.

for longer wavelengths. Two-photon absorption coefficients of $17 \pm 7$ and $14 \pm 6 \mathrm{~cm} / \mathrm{GW}$ can be derived for wavelengths of 387 and $391 \mathrm{~nm}$, which agree well with the reported values from a separate pump-probe measurement. ${ }^{4}$ The measured nonlinear refractive index versus wavelength is shown (solid circle) in Fig. 3. A maximum 25\% fluctuation in the values of $n_{2}$ was found at different sample positions. Combined with the deviation due to pulse-width fluctuation, spotsize variation, ${ }^{8}$ and fitting errors, $n_{2}$ values of $-2.9 \pm 1.2$ $\times 10^{-12},-1.2 \pm 0.5 \times 10^{-12}$, and $-0.5 \pm 0.2 \times 10^{-12} \mathrm{~cm}^{2} / \mathrm{W}$ can be obtained for wavelengths of 368,371 , and $380 \mathrm{~nm}$, respectively.

Sheik-Bahae and co-workers have derived a formula for

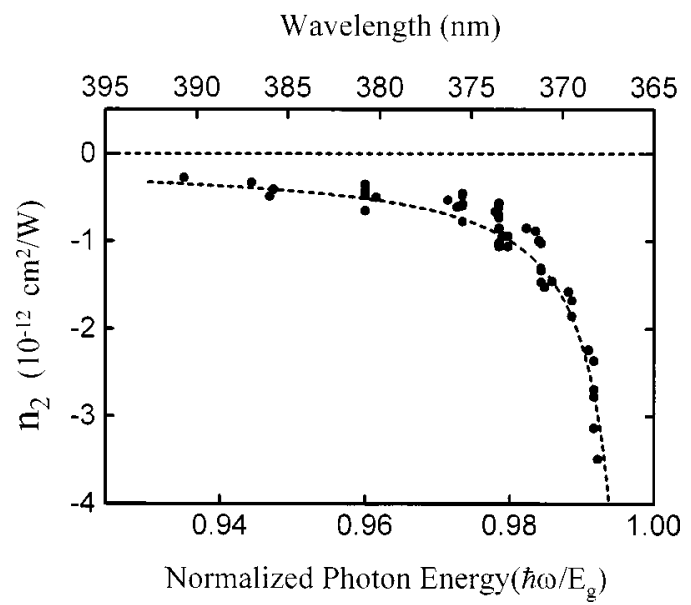

FIG. 3. Measured frequency-dependent nonlinear refractive index of GaN (solid circle). The dash line is a fitting curve governed by two-photon absorption and quadratic Stark effect. 
frequency-dependent $n_{2}$ by a two-parabolic-band model based on the Kramers-Krönig (KK) relation. ${ }^{9}$ The physical processes of two-photon absorption, Raman transitions, and ac Stark effect are considered in the model. The scaled form for nondegenerate $n_{2}$ is given by

$$
n_{2}(\mathrm{esu})=K^{\prime} \frac{\sqrt{E_{p}}}{n_{0} E_{g}^{4}} G_{2}\left(\frac{\hbar \omega}{E_{g}}\right),
$$

where the function $G_{2}$ contains the band-structure-dependent information. The value of $K^{\prime}$ predicted by theory gives $0.94 \times 10^{-8}$ when $E_{p}$ and $E_{g}$ are defined in eV. $E_{p} \approx 21 \mathrm{eV}$ for most direct-gap semiconductors. $n_{0}$ is the linear refractive index. $E_{g}$ is the band-gap energy. Previous study shows that the most significant contribution to the spectral dependence of $G_{2}$ arises from the two-photon absorption term, except close to the band edge where the quadratic Stark term becomes dominant. The functions $G_{2}\left(\hbar \omega / E_{g}\right)$ of these two processes can be found in Ref. 9. Agreement of this theory to the experimental data of semiconductors is excellent. However, the agreement for wide-band-gap materials is not as good, although general trends such as increasing nonlinearity with decreasing band-gap energy can be seen. ${ }^{10}$

A fitting curve (dash line) with $G_{2}\left(\hbar \omega / E_{g}\right)$ governed by two-photon absorption and the quadratic Stark effect is also shown in Fig. 3. The parameters used in the fitting curve are $E_{p}=21 \mathrm{eV}, E_{g}=3.39 \mathrm{eV}, n_{0}=2.7$, and $K^{\prime}=17 \times 10^{-8}$. An excellent agreement can be obtained. Although $K^{\prime}$ is 18 times larger than the theoretical value $\left(K^{\prime}=0.94 \times 10^{-8}\right)$, the nonlinear refractive indexes of $\mathrm{GaN}$ from our measurement are on the same order with the positive $n_{2}$ measured at $532 \mathrm{~nm}$ in the GaN epilayers ${ }^{2}$ and the negative $n_{2}$ measured at below-band-gap wavelengths in AlGaAs. ${ }^{11}$ Besides a large $K^{\prime}$, the distribution of the experimental data is well consistent with previous theories. ${ }^{9}$ It means that the near-band-gap nonlinear refractive index in $\mathrm{GaN}$ is primarily induced by the quadratic Stark effect. When the optical photon energy is close to the band-gap energy, the magnitude of the nonlinear refractive index will increase greatly.

Nonlinear refractive indexes are seldom reported in the UV region. $\mathrm{SiO}_{2}$ has been reported to have a positive $n_{2}$ of $4.45 \times 10^{-16} \mathrm{~cm}^{2} / \mathrm{W}\left(1.7 \times 10^{-13} \mathrm{esu}\right)$ at $249 \mathrm{~nm} .{ }^{12}$ Values of $0.69 \times 10^{-16} \mathrm{~cm}^{2} / \mathrm{W}$ for $\mathrm{MgF}_{2}$ and $7.68 \times 10^{-16} \mathrm{~cm}^{2} / \mathrm{W}$ for $\mathrm{Al}_{2} \mathrm{O}_{3}$ were previously reported at the $355 \mathrm{~nm}$ wavelength. ${ }^{13}$ Comparing $n_{2}$ of $\mathrm{GaN}$ in our measurements with these data, the below-band-gap $n_{2}$ of $\mathrm{GaN}$ is $10^{4}$-order larger than those previously reported values from other UV materials. Our studies indicate that $\mathrm{GaN}$ is not only an excellent lightemitting material, but also an excellent nonlinear material with a large negative $n_{2}$ in the near-UV region.

We have also plotted the absolute values of the measured negative effective $\beta$ observed for a wavelength shorter than $375 \mathrm{~nm}$, shown as solid diamonds in Fig. 4. The background dotted line is the measured below-band-gap absorption constant on the same thin film. The absorption constants for the band-gap energy of 3.39 and $3.43 \mathrm{eV}$ are on the order of 6000 and $28000 \mathrm{~cm}^{-1}$. Excellent agreement on the increasing trend of values toward the band-gap energy can be observed, supporting our assumption that the negative effec-

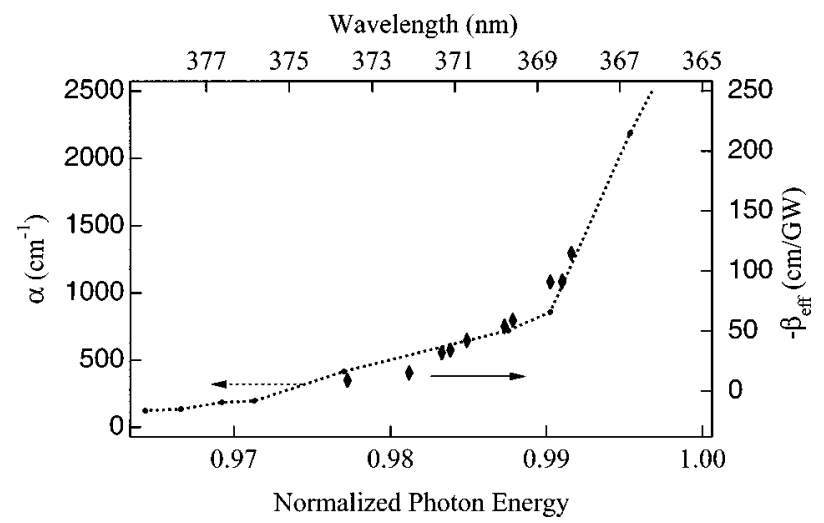

FIG. 4. Absolute values of the measured negative effective two-photon absorption constant $\beta_{\text {eff }}$ (solid diamonds) vs wavelength. The dotted line is the measured absorption constant.

tive $\beta$ should be contributed from the saturation of the bandtail states. Please notice the offset in the display of the negative effective $\beta$ due to the inherent contribution of the real two-photon absorption induced $\beta$ that is on the order of +20 $\mathrm{cm} / \mathrm{GW}$.

In summary, below-band-gap nonlinear refractive indexes of GaN were measured by a $Z$-scan technique using femtosecond UV pulses. Nonlinear refractive indexes of $-2.9 \pm 1.2 \times 10^{-12}, \quad-1.2 \pm 0.5 \times 10^{-12}, \quad$ and $-0.5 \pm 0.2$ $\times 10^{-12} \mathrm{~cm}^{2} / \mathrm{W}$ were obtained for wavelengths of 368,371 , and $380 \mathrm{~nm}$, respectively. With a large scaling constant, the wavelength distribution of the measured $n_{2}$ value is consistent with a model considering the two-photon absorption and quadratic Stark effects. ${ }^{9}$ Our study reveals the great potential of $\mathrm{GaN}$ for nonlinear UV photonic applications.

This work is sponsored by National Science Council of the R.O.C. (Taiwan) under Grant No. NSC-88-2112-M-002003 and NSC 89-2218-E-002-012.

${ }^{1}$ S. Nakamura and G. Fasol, The Blue Laser Diode (Springer, Berlin, 1997).

${ }^{2}$ B. Taheri, J. Hays, J. J. Song, and B. Goldenberg, Appl. Phys. Lett. 68, 587 (1996).

${ }^{3}$ M. Sheik-Bahae, A. A. Said, T. H. Wei, D. J. Hagan, and E. W. Van Stryland, IEEE J. Quantum Electron. 26, 760 (1990).

${ }^{4}$ C.-K. Sun, Y.-L. Huang, J.-C. Wang, S. Keller, M. P. Mack, U. K. Mishra, and S. P. DenBaars, in Technical Digest of CLEO, Baltimore, MD, Paper No. CTuI4 (1999).

${ }^{5}$ R. L. Sutherland, Handbook of Nonlinear Optics (Marcel Dekker, New York, 1996).

${ }^{6}$ R. Rangel-Rojo, S. Yamada, and H. Matsuda, Appl. Phys. Lett. 72, 1021 (1998).

${ }^{7}$ T. J. Schmidt, Y.-H. Cho, G. H. Gainer, J. J. Song, S. Keller, U. K. Mishra, and S. P. DenBaars, Appl. Phys. Lett. 73, 1892 (1998).

${ }^{8}$ F. Z. Henari, K. Cazzihi, F. Ei Akkari, and W. J. Blau, J. Appl. Phys. 78, 1373 (1995).

${ }^{9}$ M. Sheik-Bahae, D. C. Hutchings, D. J. Hagan, and E. W. Van Stryland, IEEE J. Quantum Electron. 27, 1296 (1991).

${ }^{10}$ R. Desalvo, A. A. Said, D. J. Hagan, E. W. Van Stryland, and M. SheikBahae, IEEE J. Quantum Electron. 32, 1324 (1996).

${ }^{11}$ M. J. Lagasse, K. K. Anderson, C. A. Wang, H. A. Haus, and J. G. Fujimoto, Appl. Phys. Lett. 56, 417 (1990).

${ }^{12}$ I. N. Ross, W. T. Toner, C. J. Hooker, J. R. M. Barr, and I. Coffey, J. Mod. Opt. 37, 555 (1990).

${ }^{13}$ M. Sheik-Bahae, J. R. Desalvo, A. A. Said, D. J. Hagan, and E. W. Van Stryland, Proc. SPIE 1624, 25 (1992). 